\title{
CORRESPONDENCE
}

\section{Reply to 'Side benefits sway developing nations to choose unneeded vaccines'}

\section{To the editor:}

In your report "Side benefits sway developing nations to choose unneeded vaccines" 1 , it is stated that the World Health Organization (WHO) regional office recommends that the hepatitis B vaccine only be included in routine immunization when disease prevalence exceeds $2 \%$. I would like to make the following clarification.

Everyone should be protected against hepatitis B-related diseases through vaccination. This vaccine should be offered on the basis of sound scientific information on disease burden, cost-effectiveness and safety that exist for all prevalence settings. Hepatitis B infection occurs worldwide, and it is estimated that more than 2 billion people are currently infected. Of these, about 360 million are chronically infected and at risk for serious illness and death from cirrhosis and hepatocellular carcinoma, diseases that are estimated to cause 500,000-700,000 deaths worldwide each year.
In 1992, the World Health Assembly passed Resolution 45.17, which called for Member States "to integrate cost-effective new vaccines, such as hepatitis B vaccine, into national immunization programs in countries where it is feasible." In 2004 , the WHO released its position paper on hepatitis B vaccines ${ }^{2}$. This paper stated that "routine vaccination of all infants against hepatitis $B$ virus infection should become an integral part of national immunization schedules worldwide."

The Global Alliance for Vaccines and Immunization is supporting the leastdeveloped countries in introducing hepatitis B vaccines into their national immunization programs by 2007. According to WHO/UNICEF data, 153 of $192(80 \%)$ WHO Member States reported having introduced the hepatitis $\mathrm{B}$ vaccine into their routine infant immunization schedules as of 2004. This fivefold increase in the number of countries from 1992 is the result of global advocacy, decreasing vaccine prices and the availability of resources to the poorest countries. But despite this significant progress, less than one-half of the world's infants (48\%) received three doses of the vaccine by the end of 2004 .

The availability of the vaccine to the world's children is having a significant impact on hepatitis B-related diseases. For infants born in 2003 , it is estimated that hepatitis B vaccination will prevent about 600,000 deaths during the lifespan of the vaccinated cohort ${ }^{3}$. Continued efforts to introduce this vaccine in all countries and sustained high vaccine coverage will prevent millions of deaths and deserves continued global support.

\section{Steven T. Wiersma}

Medical Officer and Hepatitis Focal Point, Department of Immunization, Vaccines and Biologicals, World Health Organization, Geneva, Switzerland.e-mail:wiersmas@who.int

1. Padma, T.V. Nat. Med. 12, 154 (2006)

2. WHO position paper. Wkly. Epidemiol. Rec. 79, 255-263 (2004).

3. Goldstein, S.T. et al. Int. J. Epidemiol. 34, 1329-1339 (2005).

\section{Reply to 'Hormone in the hot seat'}

To the editor:

In the News feature "Hormone in the hot seat" (Nat. Med. 12, 8-9; 2006), Apoorva Mandavilli reports that some of the results of the Women's Health Initiative (WHI) trial, led by the US National Institutes of Health, have been "misand overinterpreted."

The WHI studies were designed to address a very specific aim: whether or not the widely used drugs Premarin and PremPro (Premarin plus the progestin Provera) would be beneficial for postmenopausal women's heart health. The studies were a success in discrediting this form of treatment, but it is extremely unfortunate that few have bothered to look beyond the effects of estrogen on the heart. As the article says, the results have been so greatly overinterpreted and applied as to discredit all hormone replacement.
These studies were doomed from the start because they completely ignored the biology of estrogen and its receptors in the experimental design. Many experts have already noted that the apparent failure of this trial reflects the type of estrogen used (the horse urinary estrogens in Premarin, for instance), the age of the cohort studied (50-79 years) - which ignores the very narrow postmenopausal window of opportunity for positive estrogen action-and the way in which estrogen was presented continuously for years. The female brain is normally never exposed continuously to estrogen. Prolonged and uninterrupted exposure decreases the levels of estrogen receptors, leading to a loss of neuronal sensitivity to estrogen and to a decrease of its beneficial effects. Discontinuing estrogen for a few days in a cyclical manner, as normally occurs during the female reproductive cycle, would restore levels of estrogen receptor, allowing cells to become more responsive to estrogen.

Most people, including physicians and journalists, remain unaware that estrogen and progesterone have functions other than those related to reproduction. For instance, postmenopausal loss and subsequent replacement of estrogen are of great importance for the brain. During both development and adulthood, the brain is a major target of estradiol and progesterone.

In the brain, estradiol influences higher cognitive functions, mood, pain mechanisms, motor skills, susceptibility to seizures, hot flashes and sleep. The hormone appears to protect the brain from damage resulting from ischemic stroke, multiple sclerosis and Alzheimer and Parkinson diseases. Estradiol has also been shown to have a very important 